\title{
UNIVERSITYOF
}

FORWARD

THINKING

WESTMINSTER用

WestminsterResearch

http://www.westminster.ac.uk/westminsterresearch

\section{Area and Power Efficient Implementation of db4 Wavelet Filter \\ Banks for ECG Applications Using Reconfigurable Multiplier \\ Blocks}

Eminaga, Y., Coskun, A. and Kale, I.

This is a copy of the author's accepted version of a paper subsequently published in the proceedings of the 4th International Conference on Frontiers of Signal Processing (ICFSP 2018), Poitiers, France, 24 - 27 Sep 2018.

The final published version is available online at:

https://doi.org/10.1109/ICFSP.2018.8552046

(C) 2018 IEEE . Personal use of this material is permitted. Permission from IEEE must be obtained for all other uses, in any current or future media, including reprinting/republishing this material for advertising or promotional purposes, creating new collective works, for resale or redistribution to servers or lists, or reuse of any copyrighted component of this work in other works.

The WestminsterResearch online digital archive at the University of Westminster aims to make the research output of the University available to a wider audience. Copyright and Moral Rights remain with the authors and/or copyright owners.

Whilst further distribution of specific materials from within this archive is forbidden, you may freely distribute the URL of WestminsterResearch: ((http://westminsterresearch.wmin.ac.uk/)).

In case of abuse or copyright appearing without permission e-mail repository@westminster.ac.uk 


\title{
Area and Power Efficient Implementation of $d b 4$ Wavelet Filter Banks for ECG Applications using Reconfigurable Multiplier Blocks
}

\author{
Yaprak Eminaga, Adem Coskun, and Izzet Kale \\ Applied DSP and VLSI Research Group, University of Westminster, London, W1W 6UW, United Kingdom \\ Email: y.eminaga@my.westminster.ac.uk, a.coskun@westminster.ac.uk, kalei@westminster.ac.uk
}

\begin{abstract}
There is an increasing demand for wavelet-based real-time on-node signal processing in portable medical devices which raises the need for reduced hardware size, cost and power consumption. This paper presents an improved Reconfigurable Multiplier Block (ReMB) architecture for an 8-tap Daubechies wavelet filter employed in a tree structured filter bank which targets the recent Field-Programmable-Gate-Array (FPGA) technologies. The ReMB is used to replace the expensive and power hungry multiplier blocks as well as the coefficient memories required in time-multiplexed finite impulse response filter architectures. The proposed architecture is implemented on a Kintex-7 FPGA and the resource utilization, maximum operating frequency and the estimated dynamic power consumption figures are reported and compared with the literature. The results demonstrated that the proposed architecture reduces the hardware utilization by $30 \%$ and improves the power consumption by $44 \%$ in comparison to architectures with general purpose multipliers. Thus, the proposed implementation can be deployed in low-cost low-power embedded platforms for portable medical devices.
\end{abstract}

Index Terms-biosignal processing, Discrete Wavelet Transform, multiplierless, reconfigurable multiplier blocks, power analysis.

\section{INTRODUCTION}

Wearable biosignal acquisition systems with embedded digital signal processing capabilities have been a major research focus in the wireless long-term health monitoring field. The application of on-sensor signal processing is crucial due to its great potential for reducing the system power consumption, introducing robust and autonomous results, and decreasing the amount of data to be transmitted [1]. One dimensional Discrete Wavelet Transform (DWT) is extensively used in a wide range of biomedical signal processing applications including denoising, and feature extraction, and there has been a significant amount of studies in order to minimize the implementation cost and power dissipation of the DWT filter banks [2]-[4]. Most of this research is concentrated on the multiplier free design of the wavelet filters where either shift-add operations [4] or distributed arithmetic [5] is used to replace the constant multiplications i.e. multipliers. In the authors' previous work [2], an efficient multiplier free implementation of an 8-tap Daubechies $(d b 4)$ wavelet filter using a ReMB was presented and was shown that the proposed design reduced the hardware complexity by $25 \%$ in comparison to conventional fixed point implementation using a General Purpose (GP) multiplier. In this paper, a new ReMB design is proposed and implemented on an FPGA which utilizes modified and improved shiftadd networks, where the resource utilization and the power dissipation figures are presented. Section II introduces the preliminaries of the method used to design and implement the ReMB specific for 8-tap Daubechies filter coefficients. In addition, further details regarding the structure of the ReMB are shown in Section II. In Section III, resource utilization and estimation of power dissipation figures are demonstrated and compared with other $d b 4$ implementations from the open literature. Finally, Section IV presents the conclusions.

\section{METHOD}

The concept of ReMB employs efficient use of the Configurable Logic Blocks (CLBs) of the Xilinx FPGAs which are composed of Look-Up Tables (LUTs), registers, fast carrychain logic and wide multiplexers [6]. This technology is used to replace the multiple constant multiplications with more hardware and power efficient shift-add networks.

It is well known that a 1-bit full adder/subtracter (will be referred to as adder in the rest of this document) can be implemented using the dedicated carry-chain logic and an LUT to implement the remaining XOR gate. In [7], the authors proposed placement of a 2-to-1 multiplexer (2:1 mux) before a 1-bit adder where the output of the mux is connected to either one of the adder inputs. This multiplexer can be implemented inside the LUT which was used for the aforementioned XOR gate, thus no additional hardware is required. This new configuration of a 1-bit adder, adds reconfigurability to the design where two different results can be obtained with the same configuration and hardware resources. Each of the muxadder combinations are referred as 'basic structure' and is presented in Fig. 1(a). An example of possible outcomes are also presented in Fig. 1 where $S$ is the operation output, $S_{0}$ is the mux select line that can be either 0 or $1, A, B_{0}$ and $B_{1}$ are the inputs and ' $<<a$ 'represents the amount of hard-wired left shift to scale the corresponding inputs. In this particular configuration $S_{0}$ is used as the carry-in input where $S_{0}=0$ and $S_{0}=1$ lead to an addition and a subtraction operation, respectively. Of course, the output and the behaviour of the adder can be altered by making minor modifications. Further details regarding this method can be found in [7]. Although this is a very efficient concept and still can be used for replac- 
ing constant multiplications with shift-add networks, it is outof-date since it was introduced for the Xilinx 4-series FPGAs which employed 4-input LUTs. In [2], the authors extended this method for the recent FPGAs which replace 4-input LUTs with 6-input ones, that led to the replacement of $2: 1$ muxes with $3: 1$ ones for no additional cost. This modification is presented in Fig. 1(b) where either one of the mux select lines $S_{0}$ or $S_{1}$ is used for controlling the operation of the adder and three different outcomes are obtained. This simple modification adds more reconfigurability to the basic structure to reduce the hardware complexity further, especially for high filter orders. However, this method can be further improved by using 4:1 muxes which have the same hardware requirements as the 3:1 muxes but with one additional input port. A 4:1 mux requires 6 input ports where two are for the select lines and 4 for the inputs. Since the 7-series FPGAs consist of only 6-input LUTs, an extra one input will cause utilization of an additional LUT, which is not desired. Therefore, one of the inputs of the mux must be shared amongst others. Although input sharing might seem as redundant and unnecessary, it actually provides more reconfigurability to the design. This is due to the fact that the select lines of the muxes are also used for controlling the operation of the adder as addition or subtraction. This enables the full utilization of the FPGA blocks which in turn further reduces the hardware usage. Fig. 1(c) demonstrates the proposed basic structure which can achieve four different outputs with three distinct inputs as opposed to three outputs which was presented in [2].

A set of coefficients can be implemented by interconnecting
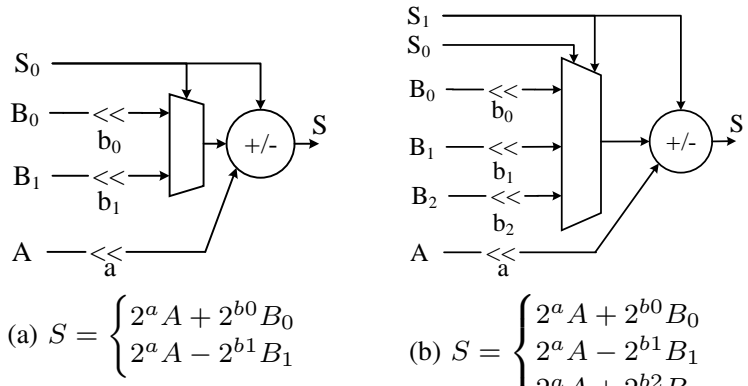

(b) $S=\left\{\begin{array}{l}2^{a} A+2^{b 0} B_{0} \\ 2^{a} A-2^{b 1} B_{1} \\ 2^{a} A+2^{b 2} B_{2}\end{array}\right.$

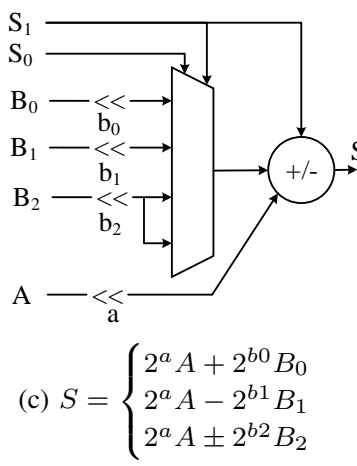

Fig. 1. Basic structures with (a) 2:1 mux [7] (b) 3:1 mux [2] and (c) 4:1 mux.
TABLE I

FIXED-POINT (11-BIT) $d b 4$ WAVELET FILTER COEFFICIENTS, THEIR ADDER DEPTHS AND SHIFT-ADD FORMAT USED TO DESIGN THE PROPOSED REMB.

\begin{tabular}{|c|c|c|c|c|}
\hline Fixed-point & $\mathbb{Z}$ & Odd & $\begin{array}{c}\text { Adder } \\
\text { Depth }\end{array}$ & Shift-Add \\
\hline \hline 0.0107421875 & 11 & 11 & 2 & $2^{2}(2+1)-1$ \\
0.033203125 & 34 & 17 & 1 & $2\left(2^{4}-1\right)$ \\
0.03125 & 32 & 1 & 0 & $2^{5}$ \\
0.1875 & 192 & 3 & 1 & $2^{6}(2+1)$ \\
0.0283203125 & 29 & 29 & 2 & $2^{2}(4+1)+\left(2^{3}+1\right)$ \\
0.630859375 & 646 & 323 & 2 & $2\left(2^{6}(4+1)+(2+1)\right)$ \\
0.71484375 & 732 & 183 & 2 & $2^{2}\left(2^{6}(2+1)-\left(2^{3}+1\right)\right)$ \\
0.23046875 & 236 & 59 & 2 & $2^{2}\left(2^{2}(16-1)-1\right)$ \\
\hline
\end{tabular}

the basic structures, given in Fig. 1(c); in chain (i.e. horizontally cascaded) and tree forms (i.e. inputs of a mux connected to the output of another basic structure). For example, cascade of two basic structures provides an output set of $16(4 \times 4)$ coefficients. Thus for a coefficient set of 8 , two basic structures is sufficient. However, the number of adders required by each coefficient is also a vital parameter while designing a ReMB which determines the minimum number of adders in each path from the input to the output of the ReMB as well as the quantity of basic structures to be used. Thus, the number of coefficients and the minimum number of adders for each coefficient must be determined.

\section{A. Design of Reconfigurable Multiplier Block for db4 filter coefficients}

The coefficient word-length plays a significant role in the design of the ReMBs since the structure of the multiplier block depends on the desired coefficient precision. Longer wordlengths result in increased number of adders and thus, higher resource utilization. On the other hand, insufficient number of bits will deteriorate the filter characteristics and operation. In order to achieve the minimum possible coefficient wordlength retaining the desired wavelet filter characteristics, the impact of the precision loss is measured by comparing the floatingpoint and fixed-point filter responses in terms of quantization noise power. Therefore, for this study, the filter coefficients are quantized to 11-bit (10 fractional bits and one sign bit) which results in $-68 \mathrm{~dB}$ of filter response mismatch and retains the filter operation as well as the scaling and wavelet functions. Prior to designing the ReMB, the aforementioned coefficients are scaled with $2^{10}$ in order to have integer $(\mathbb{Z})$ values and are listed in Table I.

The lowpass and highpass $d b 4$ filters employed both in the decomposition and reconstruction Filter Bank (FB) are power complimentary which states that both filters have the same coefficients but with alternating signs. Thus, there are only eight distinct coefficients and the same ReMB structure can be used for both filters with an additional 2:1 mux at the ReMB output to select between positive and negative coefficients. First of all, the adder depth, which is the minimum number of adders in a path from the input of the ReMB to its output 


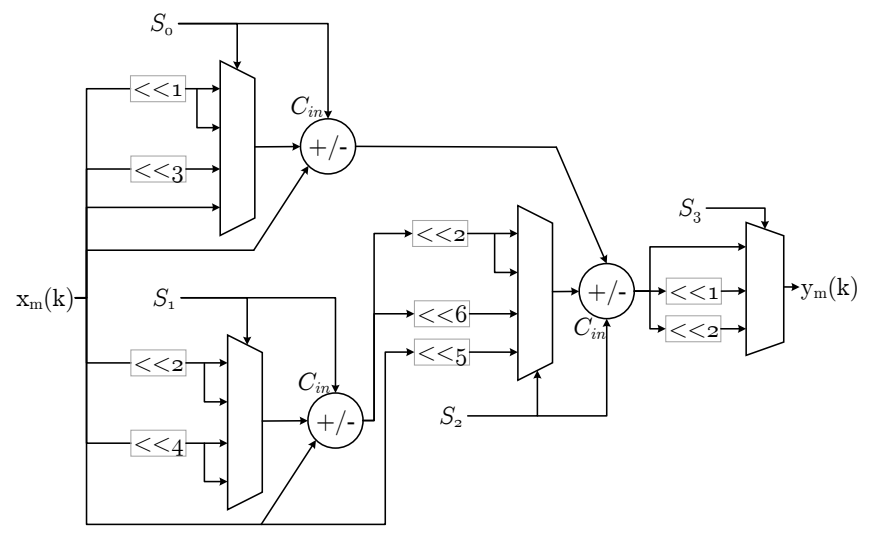

Fig. 2. The ReMB designed for $d b 4$ wavelet filters.

[7], is calculated for all eight coefficients and they are as illustrated in Table I. Although the maximum adder depth is two, a minimum of three adders are required to obtain each coefficient, thus three basic structures are interconnected and the least significant bits of the mux select lines are used as carry-ins to control the adder operation. The final structure for the ReMB is presented in Fig. 2.

\section{B. DWT Filter Bank Architecture}

Biomedical signals have frequency bands upto a few $\mathrm{kHz}$, hence they require comparably slow operating frequencies. Recent FPGAs can operate at upto a few GHz, therefore timemultiplexed architectures can be easily used and this way hardware utilization of the DWT FB can be massively reduced. A conventional time-multiplexed Tap-Delay Line (TDL) filter is composed of an input memory, a coefficient memory and a single Multiply-Accumulate unit with a GP multiplier [2], whereas in this study the GP and the coefficient memory are replaced by the proposed ReMB given in Fig. 2. Here, two tree structured 1-level analysis FBs are implemented where time-multiplexed TDL Finite Impulse Response (FIR) filter structures employing a GP multiplier and the proposed ReMB are used for the lowpass $(g(k))$ and the highpass $(h(k)) d b 4$ filters. The architecture of the FIR filter with a ReMB and the implemented FB are as shown in Fig. 3. In the case of $d b 4$ filters, the time-multiplexed TDL architecture will operate sequentially and the filter will provide an output once every eight clock cycle. The proposed ReMB produces the intermediate results of input and coefficient multiplication at each clock cycle which eliminates the need for a coefficient memory. For the implementation, quantization is not applied to the internal arithmetic of the ReMB in order to retain the highest precision. The filter output is then truncated to discard the fractional part and scaled down by $2^{-10}$. For validating the proposed structure, an 8-bit ElectroCardioGram (ECG) data obtained from the MIT-BIH Arrhythmia database is fed to the FB where the ReMB , accumulator and filter outputs are 19-, 20- and 10-bit, respectively.
TABLE II

SELECT LINE (S0:S4) VALUES FOR MULTIPLEXERS GIVEN IN FIGS.2 AND 3 TO GENERATE THE LOWPASS ANALYSIS FILTER COEFFICIENTS.

\begin{tabular}{|c|c|c|c|c|c|c|}
\hline & $\mathbb{Z}$ & $S_{0}$ & $S_{1}$ & $S_{2}$ & $S_{3}$ & $S_{4-g}$ \\
\hline \hline$h_{0}$ & -11 & 1 & 1 & 1 & 0 & 1 \\
$h_{1}$ & 34 & 0 & 0 & 1 & 1 & 1 \\
$h_{2}$ & 32 & 3 & $\mathrm{X}$ & 3 & 0 & 1 \\
$h_{3}$ & -192 & 3 & 1 & 2 & 0 & 0 \\
$h_{4}$ & -29 & 2 & 0 & 0 & 0 & 1 \\
$h_{5}$ & 646 & 0 & 0 & 2 & 1 & 0 \\
$h_{6}$ & 732 & 2 & 1 & 2 & 2 & 1 \\
$h_{7}$ & 236 & 1 & 3 & 1 & 2 & 0 \\
\hline
\end{tabular}

\section{Controller}

The controller is simply an up-counter followed by a decoder where the counter generates the address to control the input memory and the decoder decodes these addresses to generate the mux select lines. Table II presents the select line values required to generate the lowpass filter $(g(k))$ coefficients. $S_{0}: S_{3}$ corresponds to the select lines of the muxes given in Fig. 2 and $S_{4-g}$ is the mux at the output of the ReMB given in Fig. 3. Select line values $0,1,2$, and 3 choose the mux input from top to bottom, 0 and 3 selecting top and bottom input, respectively, where $X$ is a Don't Care indicating that the muxes and adders are not employed in the generation of the corresponding coefficient.

\section{HARDWARE VALIDATION AND COST AsSESSMENT}

For hardware validation, cost assessment and performance evaluation the aforementioned filter bank architectures are designed using the System Generator for DSP in the Matlab/Simulink environment and are synthesized and implemented on a Kintex-7 (xc7k325tffg900) FPGA with Vivado v16.2. In order to, validate the performance of the system, 8-bit ECG dataset from the MIT-BIH Arrhythmia database are used. The resource utilization for both filter banks are presented in Table III in terms of, LUTs, Registers, Adders and Multipliers.

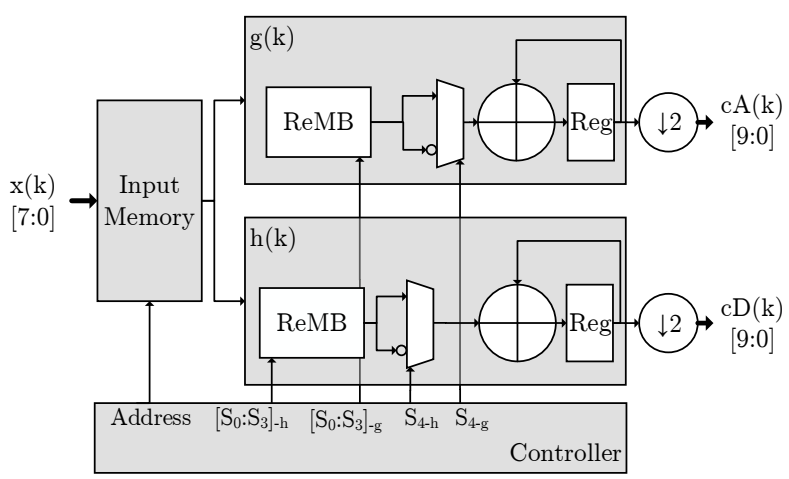

Fig. 3. One level analysis filter bank comprised of a lowpass $(g(k))$ and highpass $(h(k))$ time-multiplexed TDL filters as well as the input memory and the controller. 
TABLE III

Resource Utilization and Power Consumption of the Multiplier Free $d b 4$ Filter Bank Architectures

\begin{tabular}{|c|c|c|c|c|c|c|c|}
\hline & $\begin{array}{l}\text { Wahid [4] } \\
\left(\text { AIQ) }{ }^{1}\right.\end{array}$ & $\begin{array}{l}\text { Wahid [4] } \\
\qquad(\mathrm{FP})^{2}\end{array}$ & Longa [3] & $\begin{array}{c}\text { Hasan [8] } \\
\text { (Scheme 1) }\end{array}$ & $\begin{array}{l}\text { Hasan [8] } \\
\text { (Scheme 2) }\end{array}$ & $\mathrm{CFB}^{3}$ with GP & Proposed (ReMB) \\
\hline Architecture & Matrix & Matrix & $\mathrm{DA}^{4}$ & Lifting & Lifting & Time-multiplexed & Time-multiplexed \\
\hline Input word length (bits) & 8 & 8 & 8 & 8 & 8 & 8 & 8 \\
\hline Adders & 22 & 27 & - & 27 & 24 & 1 & 4 \\
\hline Multipliers & 0 & 0 & 0 & 0 & 0 & 1 & 0 \\
\hline LUTs & 734 & 692 & 614 & 470 & 389 & 309 & 218 \\
\hline Registers & 180 & - & - & 133 & 101 & 158 & 158 \\
\hline Max. Frequency $(\mathrm{MHz})$ & 69 & - & 149.3 & 63 & 112 & 160 & 164 \\
\hline Power $(\mathrm{mW})^{5}$ & 6.8 & 8.1 & - & - & - & 3.026 & 2.097 \\
\hline Device & Cyclone II & Cyclone II & Stratix II & Virtex-6 & Virtex-6 & Kintex-7 & Kintex-7 \\
\hline
\end{tabular}

${ }^{1}$ Algebraic Integer Quantization (AIQ) $\quad{ }^{2}$ Finite-Precision (FP) $\quad{ }^{3}$ Conventional Filter Bank (CFB) $\quad{ }^{4}$ Distributed Arithemtic (DA)

${ }^{5}$ Measured at $50 \mathrm{MHz}$

In addition, Table III presents and compares the resource utilization, maximum clock frequency and the dynamic power consumption figures (if applicable) of other multiplier free $d b 4$ analysis filter bank implementations from the open literature along with the proposed ReMB implementation. System power consumption is estimated at clock speed of $50 \mathrm{MHz}$ in order have fair comparisons with the literature and Xilinx Power Estimator tool is used for more accurate analysis. In [4], Wahid. et al. presented a matrix based AIQ mapped and a conventional fixed-point 1-level decomposition $d b 4$ filter bank architecture. The hardware cost was listed as 734 and 692 LUTs for AIQ and FP based implementation, respectively for coefficients with 10-bit precision. A more recent study by Hasan et al. [8], proposed two architectures which were lifting-based structures of the $d b 4$ wavelet filters. Here the filter coefficients were divided into lifting steps and shiftadd networks were used for implementing two 1-level decomposition FB without multipliers. The resource utilizations for both FBs were reported as 470 LUTs, 133 Registers and 389 LUTs, 101 Registers for Scheme 1 and Scheme 2, respectively. In this work, two 1-level decomposition FBs are implemented where the one employing a GP multiplier serves as a reference design. The ReMB based filter bank employs 218 LUTs and 158 registers where the GP multiplier based FB employs 309 LUTs and 158 Registers. When compared to the literature and the reference design, the proposed ReMB based FB exhibits the least hardware resources and the fastest frequency of operation. In [4], the power consumption figures were also presented for the two architectures implemented which were 6.8 and $8.1 \mathrm{~mW}$, respectively, whereas power consumption of the proposed designs are 2.097 and $3.026 \mathrm{~mW}$, respectively, where the use of ReMB improves the dynamic power consumption by $44 \%$ compared to the GP multiplier based FB and is the lowest amongst others.

\section{CONCLUSION}

In this paper an area and power efficient multiplierless filter architecture to be employed in both decomposition and reconstruction wavelet filter banks is presented. The proposed architecture is designed as a time-multiplexed TDL FIR filter in which the multiplier is replaced with a ReMB that is implemented via hard-wired shifts, adders and multiplexers. In order to evaluate resource efficiency and power consumption, the proposed architecture is implemented on a Kintex-7 FPGA and compared to a reference design implemented using a standard parallel multiplier and to the designs existing in the open literature as detailed in Table III. As the results reported in Table III demonstrate, the proposed ReMB decreases the overall hardware cost by $30 \%$ and improves the dynamic power consumption by $44 \%$ compared to a GP multiplier architecture. The low-cost and hardware efficient structure of the proposed multiplier is suitable for DWT filter banks and may be used in low-cost embedded platforms for ambulatory physiological signal monitoring and analysis.

\section{ACKNOWLEDGMENT}

The authors wish to thank the University of Westminster Faculty of Science and Technology for the PhD Studentship.

\section{REFERENCES}

[1] A. J. Casson, "Opportunities and challenges for ultra low power signal processing in wearable healthcare," in Signal Processing Conference (EUSIPCO), 2015 23rd European. IEEE, 2015, pp. 424-428.

[2] Y. Eminaga, A. Coskun, and I. Kale, "Multiplier Free Implementation of 8-tap Daubechies Wavelet Filters for Biomedical Applications," in 2017 New Generation of CAS (NGCAS). IEEE, 2017, pp. 129-132.

[3] P. Longa, A. Miri, and M. Bolic, "A flexible design of filterbank architectures for discrete wavelet transforms," in IEEE International Conference on Acoustics, Speech and Signal Processing, 2007., vol. 3. IEEE, 2007, pp. III-1441.

[4] K. A. Wahid, M. A. Islam, and S.-B. Ko, "Lossless implementation of Daubechies 8-tap wavelet transform," in IEEE International Symposium on Circuits and Systems (ISCAS), 2011. IEEE, 2011, pp. 2157-2160.

[5] A. M. Al-Haj, "Fast discrete wavelet transformation using FPGAs and distributed arithmetic," International Journal of Applied Science and Engineering, vol. 1, no. 2, pp. 160-171, 2003.

[6] Xilinx, "Series FPGAs configurable logic block," User Guide, San Jose, $C A$, vol. 1, 2016.

[7] S. S. Demirsoy, I. Kale, and A. Dempster, "Reconfigurable Multiplier Blocks: Structures, Algorithm and Applications," Circuits, Systems, and Signal Processing, vol. 26, no. 6, pp. 793-827, 2007.

[8] M. M. Hasan and K. A. Wahid, "Low-Cost Lifting Architecture and Lossless Implementation of Daubechies-8 Wavelets," IEEE Transactions on Circuits and Systems I: Regular Papers, 2018. 